\title{
Design and Experimentation of Wearable Body Sensors
}

\author{
Kiing Ing Wong \\ Curtin University \\ Malaysia
}

\section{Introduction}

The recent progress in several areas of technologies, such as microelectronic, information technology (IT) and medicine, has enable healthcare professionals to provide their services to patient remotely, without much interfere to their daily life. Researchers and scientists worldwide are therefore gaining momentum to develop a low-power, small size, wireless, wearable medicine device, or commonly referred to the body sensor. Patient is equipped with the body sensor in order to allow temporal physiological data, such as electrocardiogram (ECG or EKG), heart rate and blood pressure, to be collected. The existing telecommunication system is used to connect the patients and service providers for healthcare management and disease diagnostics purposes (Jones et al., 2010).

The implementation of body sensor into healthcare system will bring numerous advantages and will have significant impact on the current healthcare practices. For instance, quality of life of patient is improved because they do not have to lie still on the bed for a very long time in the hospital. Continuous physiological sign can be remotely collected while the patient is moving around in the hospital, at home or even at work, and immediate notification of patient deterioration can be detected and response can be made instantaneously. On the other hand, the healthcare personals do not have to take care of the patients closely and therefore will alleviate the pressure on the overburdened healthcare system.

In principle, the wireless medical telemetry device is already in place. For example, commercial Welch Allyn MiniHolter Event ECG recorder is measured $112 \mathrm{~mm} \times 78 \mathrm{~mm} \times$ $36 \mathrm{~mm}$ in size, weighted $396 \mathrm{~g}$ and very expensive. The commercial electrodes attached directly to human skin, for high quality signal collection, often risk of skin irritation. Therefore the wireless medical telmetry device is not suitable for continuous health montoring, and needless to say it is not popular amount patients and healthcare providers. The current challenges are how to integrate the existing technologies to form a cost-effective, low-power, small-size, wearable medical telemetry devices, that is best referred to as the body sensors. Although the custom-designed electronic chip using application-specific integrated circuits (ASICs) techniques able to achieve a low-power and small-size design, it shall be avoid as much as possible. This is because any ASIC chip can cost millions of dollars by the time the chip researches full production, and are not practical for many small- and medium-size companies. Therefore, the body sensors shall be designed based the lastest microcontroller technology and other commercial-off-the-shelf (COTS) electronic components (Milenkovic et al., 2006). 
It is essential for product development to have a low start-up cost; thus, it is best if open source development tools, such as the GNU GCC toolchain, the royalty free RTOS eCos and TinyOS, are used. We choose TinyOS to run on a commercial available wireless sensor network (WSN) platform for fast deplotment.

The goal of this chapter is to describe the how to fast prototype a wearable body sensor for detecting physiological data of patient. The hardware and software development of both ECG and PPG sensor is described in Section II and III, respectively. In Section IV, experiment setup, results and discussion are included. We conclude with several comments regarding the future developments of wearable PPG sensor in the final section

\section{Electrocardiograph and photoplethysmograph}

There are many types of physiological signal can be detected from human body, including electrocardiograph (ECG), photoplethysmograph (PPG), temperature, blood pressure, glucose level and patient activity. We have limited our discussion to ECG and PPG because these are the most common signals used for monitoring and disease diagnostics of cardiac patients. We also detect patient activities and positions, such as walking, sitting and lying using a wearable body sensor.

ECG is the recording of electrical activity of the heart over time, normally captured externally from the human skin. It has been invariable variable for physician to assess cardiovascular of patient for almost three decades, and it is now used for long-term health monitoring at home over periods of days, weeks and even months. A conventional ECG monitoring system requires ten electrodes attached to different places of human body and a total of twelve ECG waveforms are simultaneously are measured from the human body, are not suitable for long-term physiological monitoring. This ECG is normally collected in hospital, and the patient is closely supervised by an experienced physician while the ECG is collected. Generally, a body sensor is designed to measure ECG from only a single pair of electrodes, in order to minimize discomfort to patient during long-term monitoring. ECG obtained by the body sensor shall has five distinct waves (identified by letters $P, Q, R, S$ and T) to represent a single beat of the heart as shown in Fig. 1(a).

PPG is measured from the pulsating arterial blood, using optical technique, which is synchronized with the heart cycle. The deoxygenated hemoglobin (referring to blood pumping into the heart) and oxygenated hemoglobin (referring to blood pumping out of the heart) exhibit two different optical absorption levels, and it is measured as PPG signal, as shown in Fig 1(b).

The hear rate variability of patient, that can be measured by the variation in RR interval and PP interval (as shown in Fig 1, is very important physiological parameter for long-term health monitoring.

\section{Bio-signal front end circuits design}

The proposed wearable body sensor is a combination of a bio-signal front end circuit and a commercial wireless sensor network (WSN) platform. The bio-signal front end circuit is a custom-designed circuit to capture physiological signal from human body. While the WSN platform is commercial available that is used to control the bio-signal front end circuit. A general WSN platform is comprised of a microcontroller, an external flash memory and a wireless communication chip that are packed into a small printed circuit board (PCB). It 
therefore becomes invariable as a basis for experimentation of the capabilities of the microcontroller and other the integrated circuit (IC) chips. Two typical design of bio-signal front end circuit are included in the following section, for PPG and ECG signal detection, respectively.

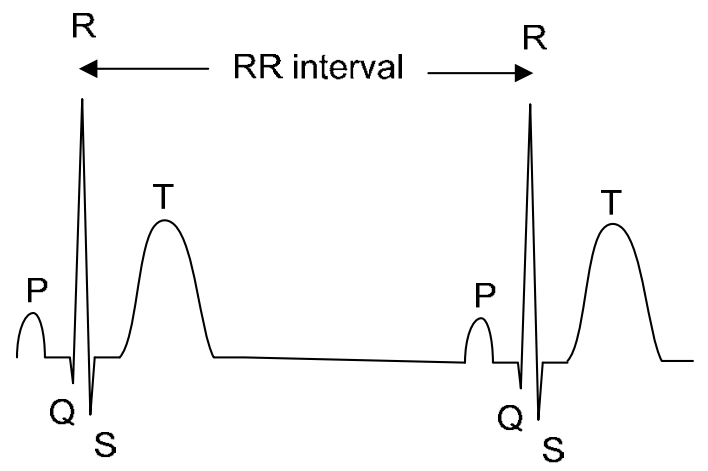

(a)

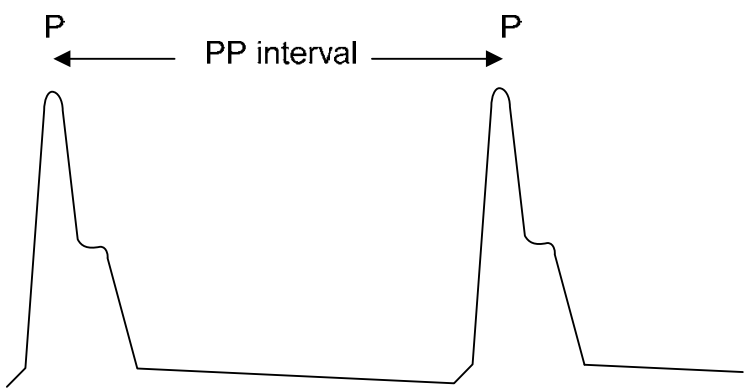

(b)

Fig. 1. (a) Diagram of idealized (a) ECG and (b) PPG.

\subsection{PPG front end circuit design}

The PPG signal detected directly from skin is comprised of a non-pulsatile part, referred to as the DC component, and a pulsatile part, the AC component. The DC component is due to light absorption by skin, tissue, venous blood, and non pulsatile arterial blood. The AC component is due to light absorption associated with pulsatile arterial blood flow, and it is only $0.1 \%$ of the total detected PPG signal. The PPG front end circuit is designed to extract the AC component only, because it contains the physiological information such as heart rate, heart rate variability and blood pressure. The circuit also design for low power and wearable operations.

\subsubsection{Transmittance versus reflectance PPG recording}

PPG measurement can operate in two different types: transmittance and reflectance. In the transmittance PPG, the light transmit through the human tissue is detected by a 
photodetector at the opposite side of the LED. While the LED and the photodetector in located in the same planar surface for the reflectance PPG measurement. The photodetector captures the light that is reflected off the human tissue. Fig. 2 shows the diagram of the transmittance and reflectance type PPG detection.

Beside the reflectance PPG can be used to detect the physiological data from human forehead, it also consumes less power as compared to the transmittance one. We are designed a reflectance PPG body sensor because it can be used to detect PPG signal from human forehead.

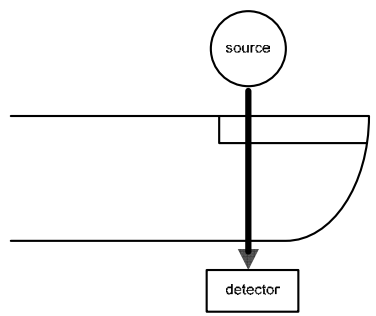

(a)

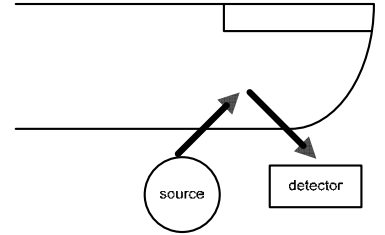

(b)

Fig. 2. (a) Diagram of (a) transmittance and (b) reflectance types PPG detection.

\subsubsection{PPG front end circuit description}

Fig. 3 shows the PPG front end circuit used in this particularly project (Wong, 2010). The $\mathrm{I} / \mathrm{O}, \mathrm{ADC}$ and DAC pins are connected to microcontroller through WSN platform. A red and an infrared surface mount LEDs (from OSA Opto Light, Germany) with peak wavelength of $600 \mathrm{~nm}$ and $875 \mathrm{~nm}$, respectively, are used in this paper. It includes an optical lens that has a narrow viewing angle of 40 degree. This is to ensure large volume of light reaches inside the human tissue while consuming a minimum amount of electrical energy. Since the LEDs consume a large amount of electrical energy when emitting light continuously. Therefore, it is switched on only for a short interval during the measurement and switched of after the measurement, through the I/O pins of microcontroller (as shown in Fig. 2).

A monolithic photodiode surface mount IC chip with on-chip transimpedance amplifier, namely OPT101 by Texas Instrument, is selected in this design. It has low leakage current errors, noise pick-up and gain peaking due to stray capacitance. It can be operated at the DC voltage at $2.7 \mathrm{~V}$, and thus it is suitable for a battery-operated body sensor design. The photodiode IC chip converts the light signals into the current signal and voltage signal, subsequently, by using the on-chip transimpedance amplifier.

The voltage signal consists of a large DC component superimposed on a small AC component. The AC component magnitude is between 0.1 and $1 \%$ of the DC component. In PPG signal condition stage, an RC filter (located between the A1 and A2 operational amplifier (op-amps)) is used to filter out the AC component. The signal output of the A2 op-amp is mainly consisted of DC component, is compared with the voltage signal output of OPT101. The A1 and A2 op-amps are used as the buffers for the input and the output signals of the RC filter. The A1 op-amp is used to amplify the AC component while block the DC component. 
The ADC0 pin of microcontroller is used to detect the total reflectance light from human tissue. The information is used to adjust the intensity of LEDs. The ADC1 pin is used to read the PPG signal.

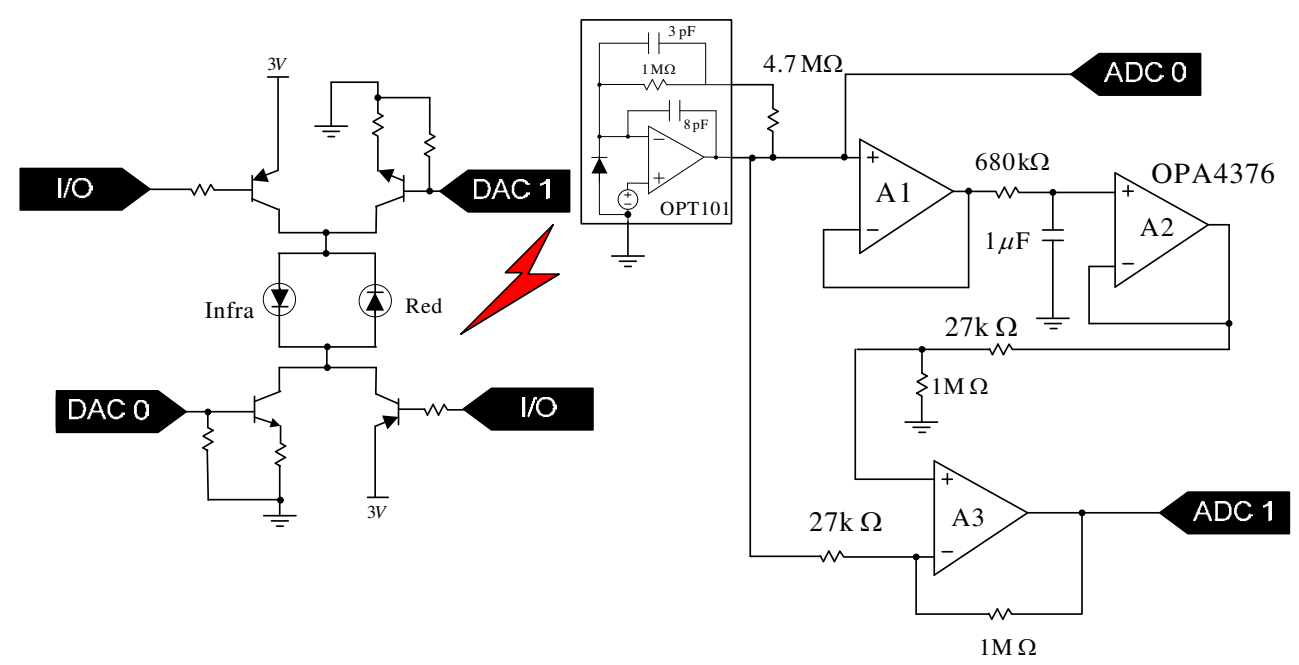

Fig. 3. Schematic of PPG front end circuit.

\subsection{ECG front end circuit design}

This section presents the ECG front end circuit design for ambulatory recordings, like longterm, wireless medical recording. The ECG front end circuit is designed based on the COTS components, without implementation of any Application Specific Integrated Circuit (ASIC) technology. This is because any application of ASIC will results in long development time and the total cost may run into millions of dollars at the stage of manufacturing.

\subsubsection{Single lead ECG recording}

A normal diagnostic ECG requires twelve leads (that requires ten electrodes to be connected to patients for detection). The patient has to lie still on the bed for a very long time, and the detection has to be prepared by an experienced physician. However, for long-term monitoring applications a single lead ECG is sufficient. This detection requires only three electrodes and provides less intrusion and skin irritation to patient.

The single lead ECG recording used for long-term, ambulatory ECG is often disturbed by various noises. The most common disturbances originated from the power line interference, electromyogram (EMG) noise, motion artefacts and baseline wander (drift). The power line frequency $(50 \mathrm{~Hz}$ or $60 \mathrm{~Hz}$ ) by ubiquitous supply lines and electrical components (such as Personal Computer) easily couple into human body through the air capacitance. In short, human body acts like a huge antenna and receive the electromagnetic wave easily. The internal interference arises from its power supply unit is not important to the ambulatory ECG equipments because these equipments are normally operated by battery.

An instrumentation amplifier (IA) which has high common-mode rejection ratio (CMRR) and low input reference noise is an important element to read the ECG signals. The power 
line interference is often presented in the form of common-mode voltage signal, $v_{c m}$, to both the input terminals of INA can be easily rejected by the device.

The ECG signal is AC coupled in the measurement stage to eliminate the DC offset potentials on the input electrodes. These DC offset potentials can be several orders higher than the ECG signal, and can be developed by several sources including respiration, motion artefacts and poor quality electrode attachment.

\subsubsection{ECG front end circuit description}

The block diagram for single lead ECG front end circuit is presented in Fig. 4. It was implemented by a general-purpose IA (INA126 by Texas Instruments) and a quad version op-amp IC (OPA4321 by Texas Instruments). The INA126 presents a CMRR of $>83 \mathrm{~dB}$, a noise of $35 \mathrm{nV} \mathrm{H}^{1 / 2}$ at $10 \mathrm{~Hz}$, an input bias current of $25 \mathrm{nA}$; these features are good enough to acquire ECG signals. The OPA4132 has four op-amps in a single IC chip therefore minimize the size of PCB design. The circuit board is powered by a $3 \mathrm{~V}$ coil cell battery.

The ECG front end circuit design follows the following steps (Wong et al, 2008):

1. Implemented AC-coupled front end at the input stage, using passive components (Spinelli et al., 2003).

2. Implemented the INA126 with high CMRR to block the common noise as much as possible.

3. Limited input stage gain to 5, in order to avoid output saturation, which is generally produced by the DC off-set voltage between the right arm and the left arm electrodes.

4. Implemented an AC-coupled at the IA stage, using the negative feedback of the A1 opamp DC output voltage (see Fig. 1). The feedback loop includes an integrator, which result in a first order high pass filter at the cut-off frequency lower than $0.5 \mathrm{~Hz}$.

5. Implemented the right-leg-driven circuit to further reduce the common-mode voltage through the A3 and A2 op-amps (Winter et al., 1983).

6. Apply high gain in the A4 op-amp stage (see Fig. 1) to boot the ECG signal by 200 times, and bring total gain by 1000 times.

7. Implement low-pass filtering in the output stage at the cut-off frequency of $40 \mathrm{~Hz}$.

\section{Wireless sensor network}

Wireless sensor network (WSN) is an emerging class of systems consisting of spatially distributed autonomous devices which have limited energy consumption and are capable to relay the data to base station through energy efficient wireless protocols. Recently, WSN has been used to monitor physical or environment conditions, such as temperature, sound, vibration, pressure and pollutants in an inter-disciplinary area between people in the electrical engineering, computer science, civil engineering and geology. For instance, WSN has been deployed on an active and hazardous volcano to provide large spatial, real-time and long period monitoring (Werner-Allen et al., 2006).

A WSN platform can be imagined as a small computer that consisted of a microcontroller, a radio transceiver, and on-board flash memory on a single printed circuit board (PCB). Fig. 5. shows a block diagram of a general WSN platform. The WSN incorporates an expansion connector to wire to external sensor circuitry and power supply. It is designed to operate at low DC voltage from battery.

The WSN platform is available commercially in different forms and from many different companies. Some of the latest available WSN platforms are listed in Table 1. Most of the 
WSN platform utilize the $2.4 \mathrm{GHz}, 915 \mathrm{MHz}$ and $868 \mathrm{MHz}$ license free industrial, scientific and medical (ISM) radio bands and operates on TinyOS operating system (TinyOS ,n.d.). TinyNode 586 utilizes sub-GHz $(868 \mathrm{MHz})$ radio band to offers better penetration of lower frequency, resulting in longer communication ranges but lower data rates as compared to the $2.4 \mathrm{GHz}$ WSN platforms.

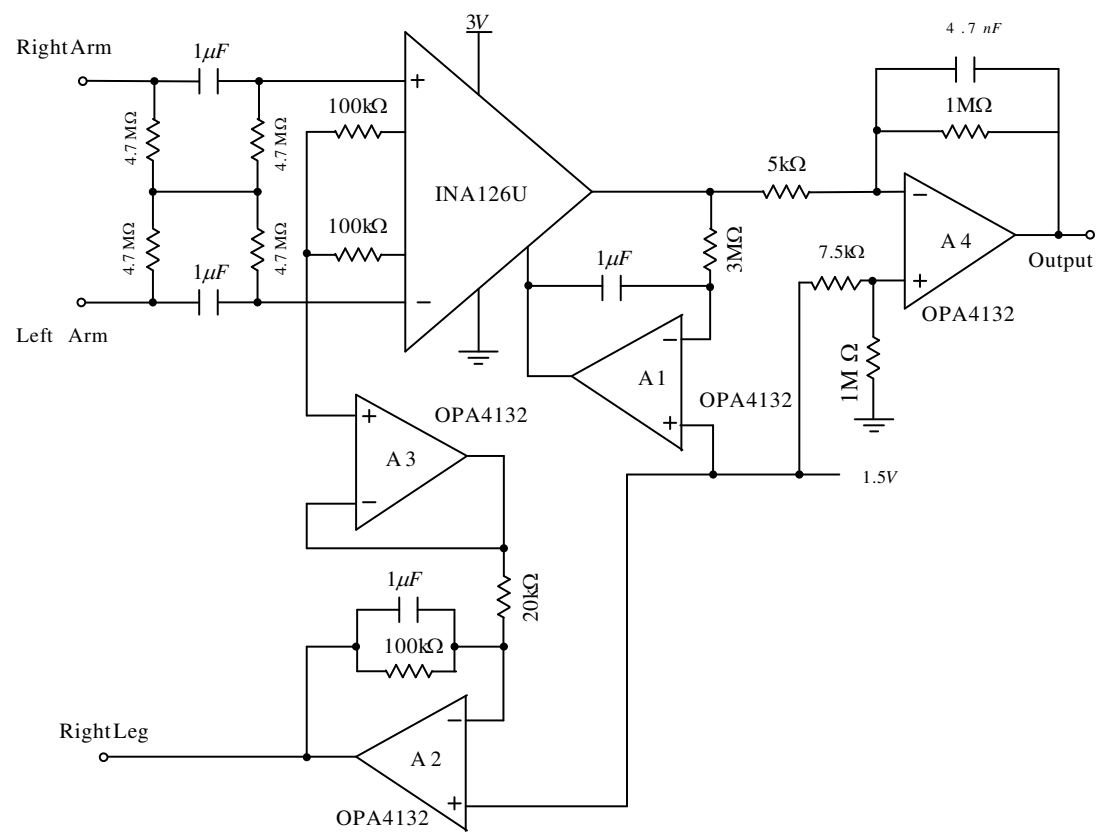

Fig. 4. Schematic of ECG front end circuit.

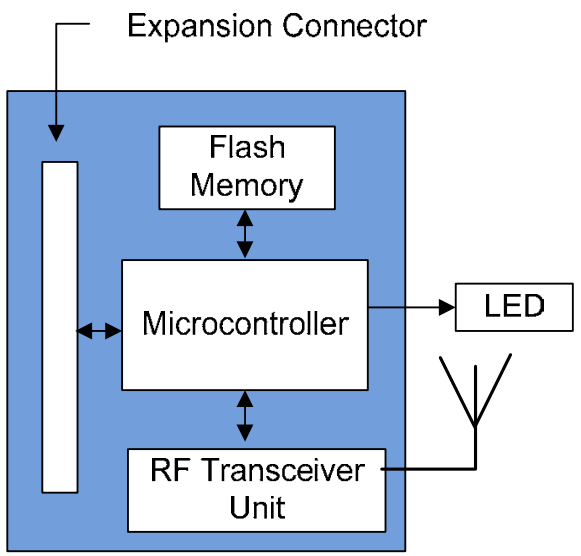

Fig. 5. Block diagram of WSN platform. 


\begin{tabular}{|c|c|c|c|c|c|}
\hline Name & TelosB & IMote2 & Mulle 3.1v & $\begin{array}{c}\text { TinyNode } \\
586\end{array}$ & Z1 \\
\hline Company & MEMSIC & MEMSIC & EISTEC AB & TinyNode & Zolertia \\
\hline $\begin{array}{c}\text { Microcontrolle } \\
\mathrm{r}\end{array}$ & $\begin{array}{c}\text { Texas } \\
\text { Instruments } \\
\text { MSP430F1611 }\end{array}$ & $\begin{array}{c}\text { Marvell } \\
\text { PXA271 }\end{array}$ & $\begin{array}{c}\text { Renesas } \\
\text { M16C }\end{array}$ & $\begin{array}{c}\text { Texas } \\
\text { Instruments } \\
\text { MSP430F1611 }\end{array}$ & $\begin{array}{c}\text { Texas } \\
\text { Instruments } \\
\text { MSP430F }\end{array}$ \\
\hline Transceiver & $\begin{array}{c}\text { TI Chipcon } \\
2.4 \mathrm{GHz} \text { IEEE } \\
802.15 .4\end{array}$ & $\begin{array}{c}\text { TI Chipcon } \\
\text { CC2420, IEEE } \\
802.15 .4\end{array}$ & $\begin{array}{c}\text { Mitsumi } \\
\text { Bluetooth } \\
\text { Module }\end{array}$ & $\begin{array}{c}\text { Xemic } \\
\text { XE1205 868 } \\
\text { MHz }\end{array}$ & $\begin{array}{c}\text { TI Chipcon } \\
\text { CC2420, IEEE } \\
802.15 .4\end{array}$ \\
\hline $\begin{array}{c}\text { Program } \\
\text { flash+Data } \\
\text { RAM }\end{array}$ & $48 \mathrm{~KB}+10 \mathrm{~KB}$ & $32 \mathrm{MB}+256 \mathrm{kB}$ & $384 \mathrm{~KB}+31 \mathrm{~KB}$ & $48 \mathrm{~KB}+10 \mathrm{~KB}$ & $92 \mathrm{~KB}+8 \mathrm{~KB}$ \\
\hline $\begin{array}{c}\text { External } \\
\text { memory }\end{array}$ & $\begin{array}{c}\text { ST M25P } \\
1 \mathrm{MB}\end{array}$ & $\begin{array}{c}\text { ST M25P } \\
32 \mathrm{MB}\end{array}$ & $\begin{array}{c}\text { Atmel } \\
\text { AT2DB161D } \\
2 \mathrm{MB}\end{array}$ & $\begin{array}{c}\text { Atmel } \\
\text { AT45DB } \\
512 K B\end{array}$ & $\begin{array}{c}\text { ST M25P } \\
2 \mathrm{MB}\end{array}$ \\
\hline $\begin{array}{c}\text { Programming } \\
\text { TinyOS, }\end{array}$ & TinyOS & $\mathrm{C}$ & TinyOS & $\begin{array}{c}\text { TinyOS, } \\
\text { Contiki }\end{array}$ \\
\hline $\begin{array}{c}\text { Dimension } \\
(\mathrm{mm})\end{array}$ & $65 \times 31 \times 6$ & $36 \times 38 \times 9$ & $23.2 \times 23.3 \times 5$ & $30 \times 40$ & $56 \times 34$ \\
\hline
\end{tabular}

Table. 1. List of commercial WSN platforms.

\section{Wearable body sensor prototype}

A commercial available wireless sensor networks (WSN) platform is offer integrated with a bio-signal front end circuit for prototyping a new body sensor. The WSN platform is lowcost, light-weighted and low-power electronic device, therefore make the physical size small and easy to wear. The bio-signal front end circuit is used to condition the physical physiological data, such as ECG and PPG signals, so that it is suitable to be read by analogto-digital (ADC) converter of microcontroller.

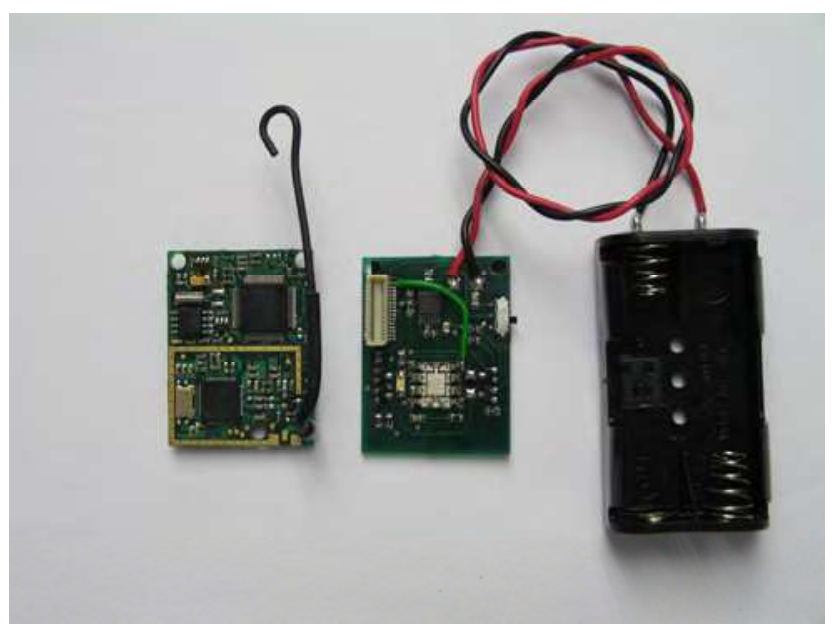

Fig. 6. Construction of PPG body sensor that consisted of a WSN device (left), an PPG front end circuit board (centre) and a battery holder (right). 


\subsection{PPG body sensor.}

All the electronics components are assembled into a $30 \mathrm{~mm} \times 40 \mathrm{~mm}$ board to achieve the PPG front end circuit. The circuit is stacked to the bottom of a WSN node to function as a PPG body sensor. The OPA4316 IC (with $0.635 \mathrm{~mm}$ pitch size) and the passive components (with $1.6 \mathrm{~mm} \times 0.8 \mathrm{~mm}$ dimension) were used in the design. The PPG front end circuit design was sent to professional PCB manufacturer, and all the electronic components were hand solder. Fig. 6 shows the construction of PPG body sensor.

\subsection{ECG body sensor.}

Fig. 6 (a) shows the construction of the ECG body sensor that consisted of three components: an ECG electrode attachment, a custom made ECG front end circuit board and the TinyNode 586 WSN platform. The ECG electrodes were placed in close proximity (as shown in Fig. 6(b)), in order to reduce the size of patient attachment and be more comfortable to wear. The surface mount technology (SMT) INA126 (with $1.27 \mathrm{~mm}$ pitch size), and the 1206

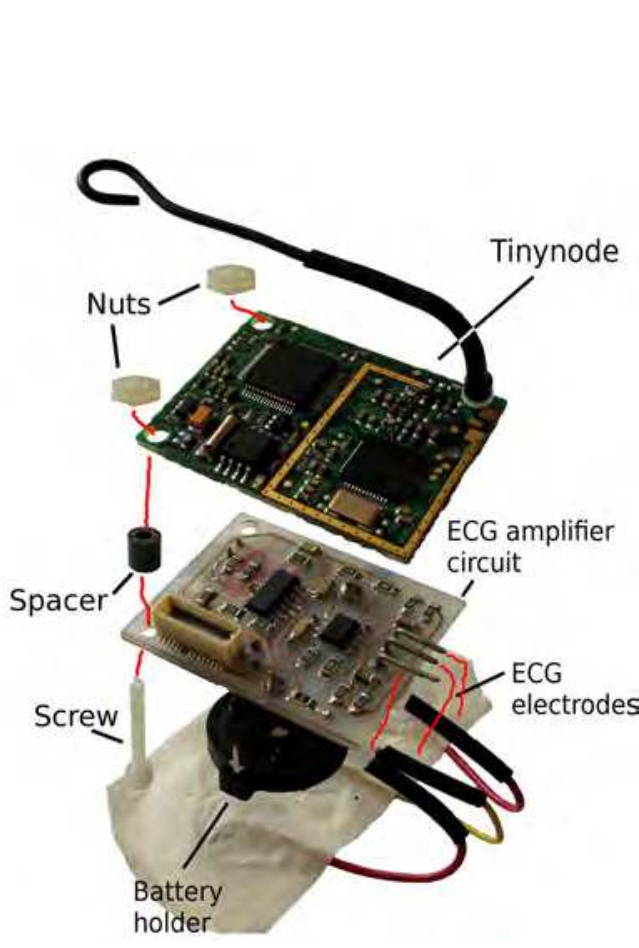

(a)

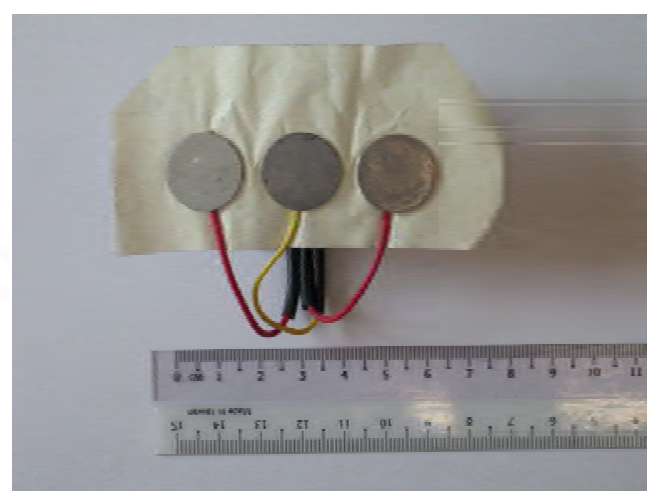

(b)

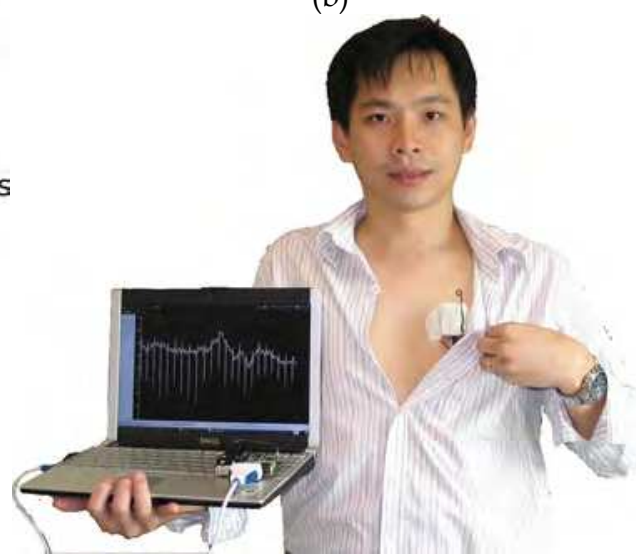

(c)

Fig. 7. (a) Construction of ECG body sensor, (b) three electrodes connected in close proximity and (c) Patient is attached with the ECG body sensor and data is transmitted tp PC for display. 
SMT resistor and capacitor (with $3.2 \mathrm{~mm} \times 1.6 \mathrm{~mm}$ dimensions) were used in the ECG front end circuit design. The PCB was prototyped using a commercial PCB milling machine in university.

The size of the IC components and the passive components (selected for the ECG front end circuit design) were considered large, even for hand soldering. If we use PCB manufactured by professional company, we can early hand solder the IC components with $0.635 \mathrm{~mm}$ pitch size and the passive components with $1.6 \mathrm{~mm} \times 0.8 \mathrm{~mm}$ dimension (see Fig.6).

\section{Software implementation}

One of the main reasons of choosing a commercial WSN platform for hardware experimentation is that the WSN platform is supported by TinyOS (tiny operating system). TinyOS is a small, open source, energy efficient, software operating system first developed by UC Berkeley, USA. It features a component-based architecture to promote code reuse and enables rapid implementation. The TinyOS components are divided into three distinctive layers. Most of the components are configured from low level components to reflect its functionality clearly, by hiding the hardware setting. The application designers are allowed to customize their applications by choosing the essential components, therefore achieve minimum code size. The TinyOS components include the data acquisition, flash memory access, wireless communication protocols, and task scheduling.

The TinyOS-based application is built on a nesC (network embedded system C) dialect, using the components that follow the event driven operations (TinyOS, n.d.). Like hardware operations, a TinyOS component commands (or signals) a function is non-blocking, or is returned immediately. The component issues an event (or callback), at some point later, after the completion of function of the component.

\section{Experimentation of wearable body sensors}

Fig. 8 shows an array of wearable body sensor attached to different part of human body, in order to obtain ECG, PPG and patient activity information. A MEMS accelerometer is interfacing with the WSN platform to capture activity information. Real-time recording of body accelerations is important to access the activity levels and relate it to ECG and PPG o patients for the diagnosis as well as management of disease. The activity information can also be used to detect fall of solitary elderly patient whom live alone in the apartment (Abbate et al., 2010).

Fall among aging population are common since they are likely to suffer from functional disorder of the body due to aging and other diseases such as hypertension, myocardial infarction and cerebral apoplexy. It should not be taken lightly because the outcome of the fall may result injuries ranges from bruises, hip fractures to even be fatal. The injury levels may reduce to minimum by prompt reaction to the fall even, once the fall has been detected.

A base station, which has similar radio chip as the wearable body sensor node, is attached to a PC to receive data and relay it to PC for display and storage.

\subsection{Experimental results of PPG body sensor}

The PPG signal was detected from human forehead, the PPG signal was sample at $200 \mathrm{~Hz}$ and represented in 12 digital bits resolution. A total of eleven samples were accumulated in 
PPG body sensor before it is radioed to the PC. This is to fully utilize the bandwidth and minimize the power consumption of wearable body sensor.
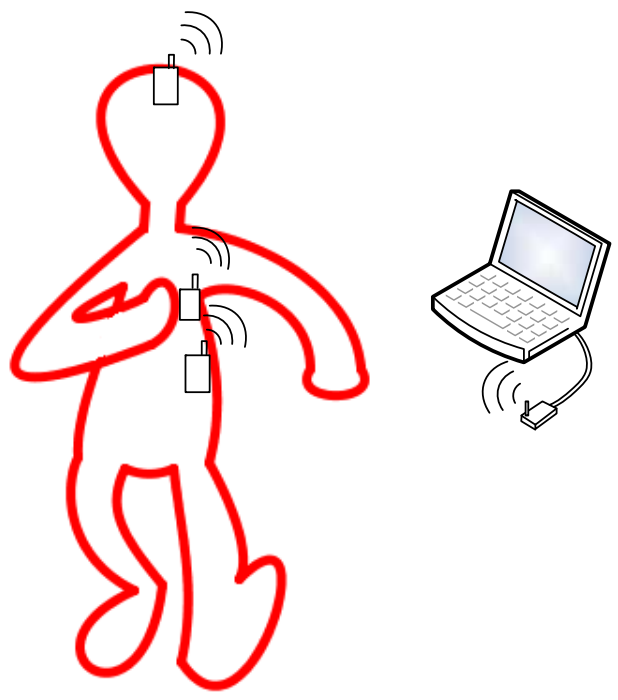

Fig. 8. (a) PPG, ECG and motion information are detected from the forehead, the chest and waist of human body by using a array of wearable body sensor, respectively. The data are radio to PC for display and storage, through a base station.

\subsubsection{Noise issues}

From the experiments, it was found that the PPG body sensor is very sensitive to wide range of noises, including the ambient light, pressure applied on body part, body temperature and motion induced artifact. Our current PPG body sensor is only suitable to be used in the laboratory environment. For instance, the casing design is not good enough to resist the out door light intensity, and very difficult to achieve the optimal pressure on between the sensor and the human forehead.

Fig. 9 (a) shows the PPG signals displayed on PC screen in the first hours, and Fig. 9 (b) shows after few hours in the air-condition office. The result shows the PPG signal amplitude decreases when the body temperature drops.

\subsubsection{Energy consumption budget}

Fig. 10 shows that the maximum current conduct is $27 \mathrm{~mA}$ (or $111 \mathrm{~mW}$ ) when the microcontroller and radio communication chip are active. The LED is turned on for PPG signal reading and turned off after the reading. Figure 4 also show that the processing time for a single PPG sample was measured at $1.25 \mathrm{~ms}$ ( $25 \%$ of its processing power), and therefore it has plenty of time before the next input sample. However, the processing rate was limited by the radio transmission strategy.

From the experiments, it was found that the PPG body sensor is very sensitive to wide range of noises, including the ambient light, pressure applied on body part, body temperature and motion induced artifact. Our current PPG body sensor is only suitable to be used in the 
laboratory environment. For instance, the casing design is not good enough to resist the out door light intensity, and very difficult to achieve the optimal pressure on between the sensor and the human forehead.

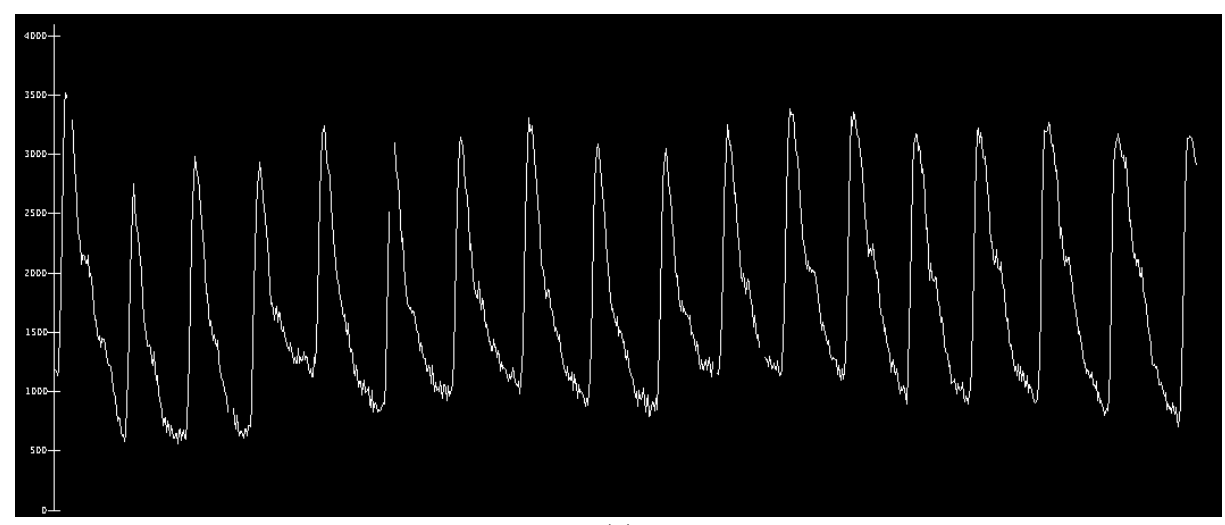

(a)

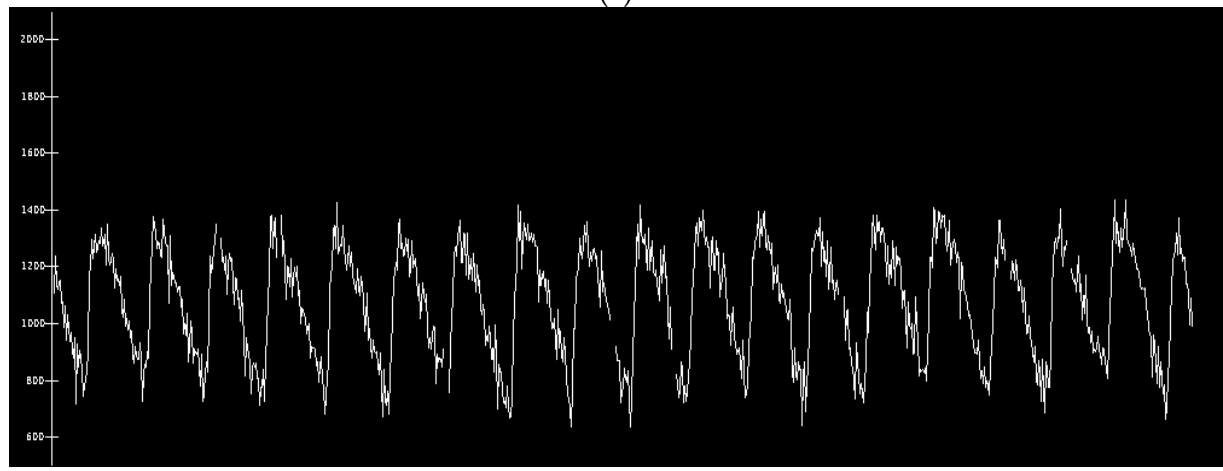

(b)

Fig. 9. PC displays of the raw PPG signals of human body received from wireless channel (a) in the first hour and (b) after few hours in air-condiction office.

\subsection{Experimental results of ECG body sensor}

The ECG sensor is taped on the chest of human body, where the signal is transmitted to a PC for display, further processing and storage. The ECG is sampled at $200 \mathrm{~Hz}$ (or 200 Sample/second) and represented in 12-bit resolution in the ECG sensor. The test human is allowed to move around while ECG is recorded.

Fig.10 shows the raw ECG waveforms produced using the Matlab software package. It shows the $\mathrm{R}$ peak is clearly detectable. The $50 \mathrm{~Hz}$ power line interference is also detected in ECG.

\subsection{Experimental results of motion body sensor}

The real-time motion information of an early patient is detected by using a motion body sensor attached to the right waist (see Fig 7). The three axis acceleration signals are read by 
the sensor at $30 \mathrm{~Hz}$ sample rate. The acceleration signals are transmitted wirelessly to PC for data processing and visualization.

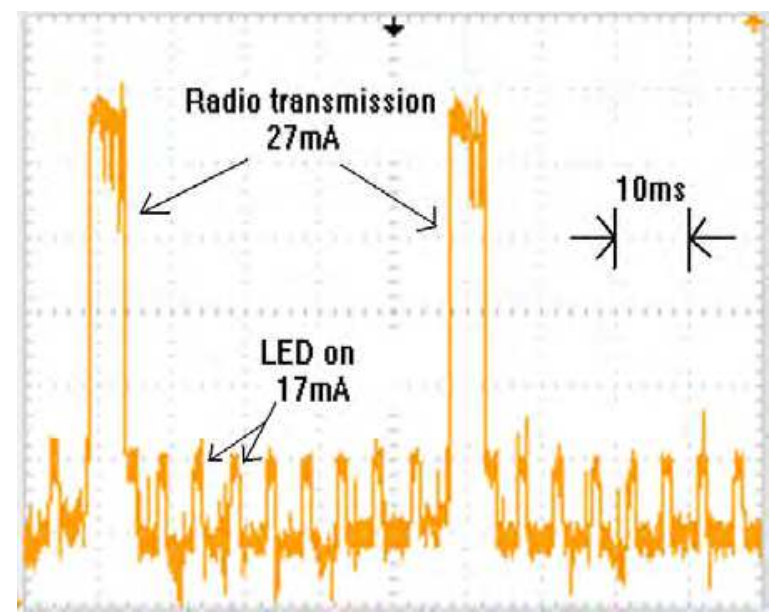

Fig. 10. Oscilloscope display of electric current values of PG body sensor.
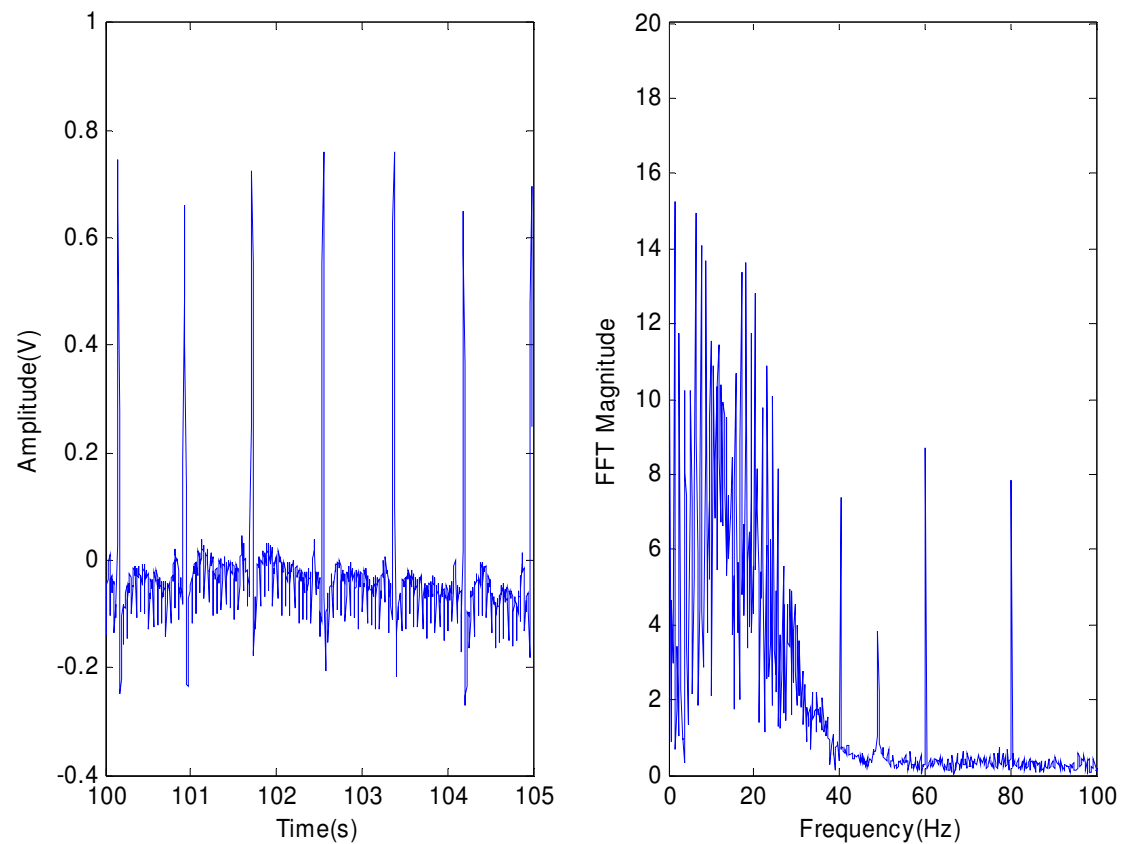

Fig. 11. (a) ECG signal and (b) its FFT magnitude response. 
In this experiment, a healthy personal was used to simulate various activities of an early patient such as sitting, walking, falling and lying on the floor. Fig. 11 shows the wireless motion information displayed on PC. The tested subject was sitting, walking, going up the stairs, coming down the stairs, walking again and suddenly fall. When lying on the ground, the subject was first lying with face down, then turned to right, face up and finally turned to left. This activity is to capture the orientation of the subject while lying on the ground.

The accelerating signals are determined using the linear equation as below:

$$
\text { Accelerating_Signal }(g)=(\text { Vout }- \text { Voff }) / S
$$

where $V_{\text {out }}, V_{\text {off }}$ and $S$ are the ADC sample data, offset voltage and sensitivity of the accelerometer. The sensitivity and offset data are obtained from the manufacturer data sheet.

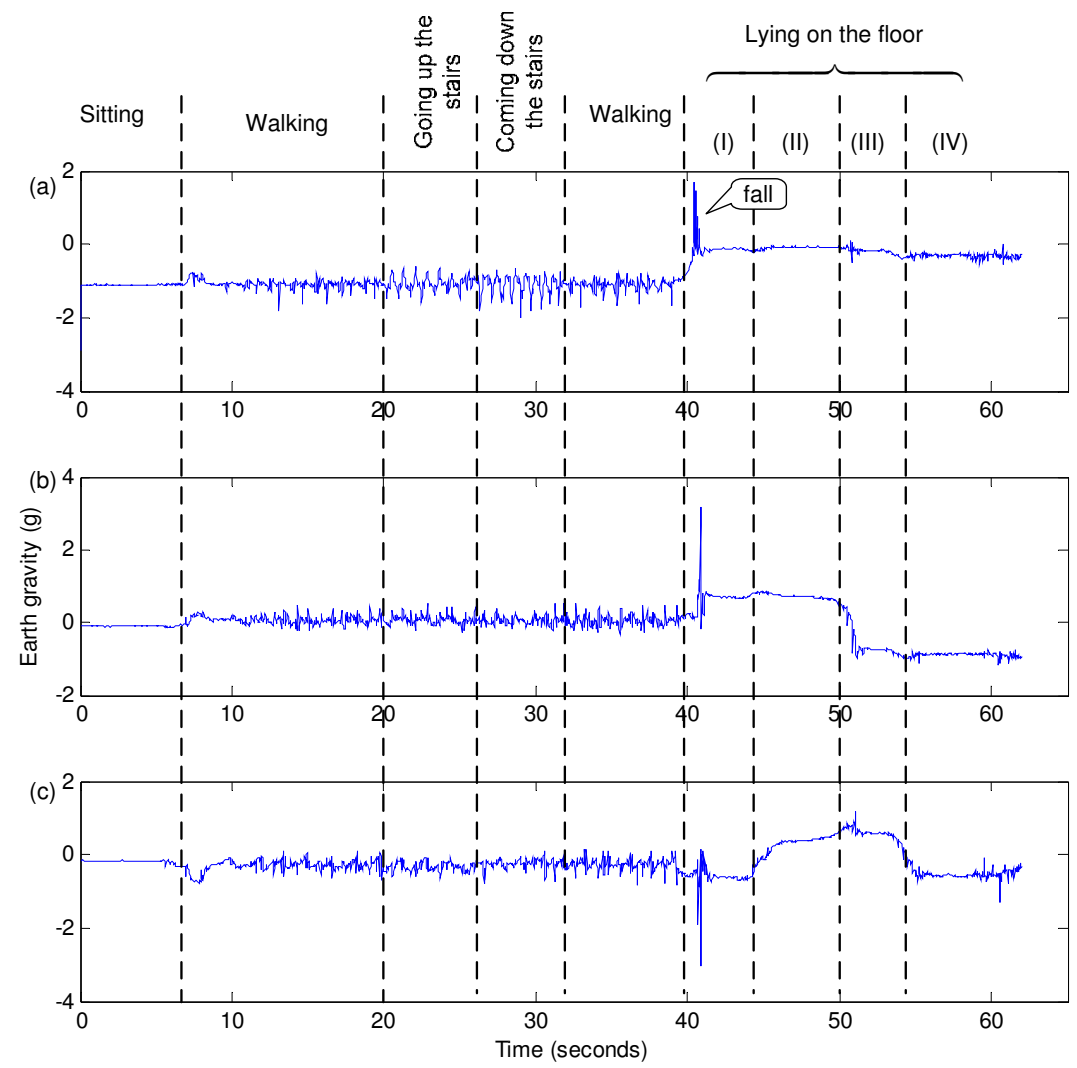

Fig. 12. PC display of $X, Y$ and $Z$ accelerating signals for (a), (b) and (c), respectively. Section (I), (II), (III) and (IV) shows the orientation of tested subject while lying on the floor with face down, left, up and right, respectively. 
As can be seen in Fig. 12, accelerating signal for all axes has the higher magnitude at the moment of falling. While the patient is lying on the floor, the accelerating signal of $x$ axis has zero magnitude. It did not change when the patient was flipping on the floor. It is aligned with the gravity. Therefore, the simple experiment had shown the technical feasibility of fall detection and the subsequent checking of the patient's orientation.

\section{Conclusion}

In this chapter, the wearable body sensors are deployed for physiological data and activity levels monitoring of patient. The wearable body sensors are designed based on a commercial available, TinyOS supported WSN platform. The WSN platform is feasible for testing the new idea in short time and at low cost, due to its intrinsic capabilities such as low power consumption, small physical size and wireless functionality.

The devices are capable of amplifying, sampling, processing and transmitting physiological data to base station wirelessly.

\section{Acknowledgment}

This work is funded by the Malaysian Ministry of Science, Technology and Innovation (MOSTI) under Project No.: 01-02-07-SF0010.

\section{References}

Abbate, S; Avvenuti, M.; Corsini, P.; Light, J. \& Vecchi, A. (2010). Monitoring of Human Movements for Fall Detection and Activities Recognition in Elderly Care Using Wireless Sensor Network : a Survey, In: Wireless Sensor Networks: Application-Centric Design, Merrett, G.V.; Tan \& Y.K., pp. 1-20, Publisher: InTech, ISBN 978-953-307321-7

Jones, V.; Gay, V. \& Leijdekkers, P. (2010). Body Sensor Networks for Mobile Health Monitoring: Experience in Europe and Australia. Proceeding of the $4^{\text {th }}$ International Conference on Digital Society (ECDS 2010), pp. 204-209, ISBN 978-1-4244-5805-9, St. Maarten, Feb 10-16, 2010

Milenkovic, A.; Otto, C. \& J. Emil. (2006). Wireless Sensor Networks for Personal Health Monitoring: Issues and Implementation. Computer Communication, Vol. 29, No. 1314, pp 2521-2533

Spinelli, E.M.; Pallas-Areny, R.; Mayosk, M.A. (2003) AC-coupled Front-End for Biopotential Measurements. IEEE Transactions on Biomedical Engineering, Vol.50, No.3, (March 2003), pp. 391-395, ISSN 00018-9294

TinyOS (n.d.) http:/ / www.tinyos.net

Werner-Allen, G.; Lorincz, K. ; Ruiz, M. ; Marcillo, O. ; Johnson, J. ; Lees, J. \& Welsh, M. (2006). Deploying a Wireless Sensor Network on an Active Volcano, IEEE Internet Computing, Vol.10, No.2, pp. 18-25, ISSN 1089-7801

Wong, K.I. \& Ho, M.M.S. (2008). Wearable Biosignal Monitoring Nodes for Real-time Electrocardiogram and Motion Measurement, Proceedings of the $5^{\text {th }}$ International Workshop on Wearable and Implantable body Sensor Networks (BSN2008), pp. 190-193, ISBN 978-1-4244-2252-4, Hong Kong, China, June 1-3, 2008 
Wong, K.I. (2010). Rapid Prototyping of a Low-Power, Wireless, Reflectance, Photoplethysmography System, Proceedings of the $8^{\text {th }}$ International Workshop on Wearable and Implantable body Sensor Networks (BSN2010), pp. 47-51, ISBN 978-07695-4065-8, Singapore, Singapore, June 7-9, 2010

Winter, B.B. \& Webster, J.G. (1983) Driven-Right-Leg Circuit Design. IEEE Transactions on Biomedical Engineering, Vol.30, No.1, (January 1983), pp. 62-66, ISSN 0018-9294 


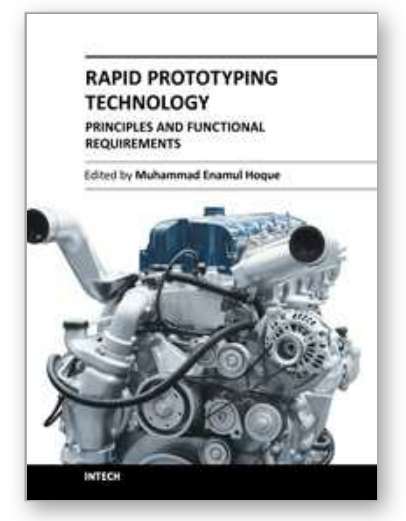

\section{Rapid Prototyping Technology - Principles and Functional Requirements}

Edited by Dr. M. Hoque

ISBN 978-953-307-970-7

Hard cover, 392 pages

Publisher InTech

Published online 26, September, 2011

Published in print edition September, 2011

Modern engineering often deals with customized design that requires easy, low-cost and rapid fabrication. Rapid prototyping (RP) is a popular technology that enables quick and easy fabrication of customized forms/objects directly from computer aided design (CAD) model. The needs for quick product development, decreased time to market, and highly customized and low quantity parts are driving the demand for RP technology. Today, RP technology also known as solid freeform fabrication (SFF) or desktop manufacturing (DM) or layer manufacturing (LM) is regarded as an efficient tool to bring the product concept into the product realization rapidly. Though all the RP technologies are additive they are still different from each other in the way of building layers and/or nature of building materials. This book delivers up-to-date information about RP technology focusing on the overview of the principles, functional requirements, design constraints etc. of specific technology.

\section{How to reference}

In order to correctly reference this scholarly work, feel free to copy and paste the following:

Kiing Ing Wong (2011). Design and Experimentation of Wearable Body Sensors, Rapid Prototyping Technology - Principles and Functional Requirements, Dr. M. Hoque (Ed.), ISBN: 978-953-307-970-7, InTech, Available from: http://www.intechopen.com/books/rapid-prototyping-technology-principles-and-functionalrequirements/design-and-experimentation-of-wearable-body-sensors

\section{INTECH}

open science | open minds

\author{
InTech Europe \\ University Campus STeP Ri \\ Slavka Krautzeka 83/A \\ 51000 Rijeka, Croatia \\ Phone: +385 (51) 770447 \\ Fax: +385 (51) 686166 \\ www.intechopen.com
}

\author{
InTech China \\ Unit 405, Office Block, Hotel Equatorial Shanghai \\ No.65, Yan An Road (West), Shanghai, 200040, China \\ 中国上海市延安西路65号上海国际贵都大饭店办公楼405单元 \\ Phone: +86-21-62489820 \\ Fax: +86-21-62489821
}


(C) 2011 The Author(s). Licensee IntechOpen. This chapter is distributed under the terms of the Creative Commons Attribution-NonCommercialShareAlike-3.0 License, which permits use, distribution and reproduction for non-commercial purposes, provided the original is properly cited and derivative works building on this content are distributed under the same license. 